\title{
5 Year-old with behavior change after febrile illness
}

\author{
Jerry W. Snow ${ }^{\mathrm{a}}$ and L.M. Tormoehlen ${ }^{\mathrm{b}}$
}

a Department of Emergency Medicine and Division of Medical Toxicology Carolinas Medical Center-Main, Charlotte, NC, United States

b Department of Neurology Indiana University School of Medicine, Indianapolis, IN, United States

5 year-old previously healthy boy presented to the emergency department for behavior change. He was diagnosed 10 days prior to with a right otitis media and started on amoxicillin. At that time, the patient did not have any URI (upper respiratory infections) symptoms, cough, or congestion. He had not received any recent immunizations. He had persistent fever for more than 7 days, so was switched to Augmentin. Three days prior to presentation his parents noticed a change in his behavior. He began becoming less interested in things that were normally very exciting to him including playing on the beach during vacation. On the day prior to presentation his behavior became more abnormal. He became much more listless and was very uninterested in playing or interacting with his family. He also had a noticeable change in his speech. He was having difficulties with word finding and would frequently start a sentence but would look confused and stop speaking after 2-4 words. His change in speech was very concerning to the parents as they described him as typically very verbal with above average language skills/development. He also experienced bowel incontinence on the day of presentation. There was no family history of trauma, or concern for ingestion. He had not had any known sick contacts.

\section{Physical examination}

Temperature Oral (F).

98.9 F.

This is the author's manuscript of the article published in final edited form as:

Snow, J. W., \& Tormoehlen, L. M. (2016). 5 Year-old with behavior change after febrile illness. Visual Journal of Emergency Medicine, 5, 44-45. https://doi.org/10.1016/j.visj.2016.06.002 
Peripheral Pulse Rate.

110 BPM.

Respiratory Rate.

26 breaths/minute.

Oxygen Saturation.

$100 \%$.

General : Alert, Not ill-appearing,

Skin : Intact, no rash.

Head : Atraumatic.

Neck : Supple, No enlarged nodes,

Eye : Pupils are equal, round and reactive to light; extraocular movements are intact, normal conjunctiva.

Ears, nose, \& throat : Tympanic membranes clear, oral mucosa moist, no pharyngeal erythema or exudate.

Cardiovascular : Regular rate and rhythm, No murmur.

Respiratory : Lungs are clear to auscultation, equal.

Gastrointestinal : Soft, Nontender, Non-distended.

Musculoskeletal : Normal ROM, normal strength.

Neurological : CN II-XII intact, normal sensory observed, gait is unsteady, but otherwise normal; some difficulty with fine motor tasks. 
The differential included viral encephalitis, seizure, ingestion, post-viral demyelinating process, intracranial mass, and stroke

He was admitted to inpatient team. Initial CBC, CMP, UA, and UDS were unremarkable. Neurology was consulted and recommended routine EEG, MRI and LP with sedation. EEG did not reveal any seizurelike activity. MRI revealed multiple scattered areas of abnormal T2 hyperintensity involving the cortex and subcortical white matter of both cerebral hemispheres (Figs. 1-3). 


\section{References}

1. Tenembaum Silvia, et al: Acute disseminated encephalomyelitis. Neurology 2007; 68: pp. S23-S36

2. Noorbakhsh Farshid, et al: Acute disseminated encephalomyelitis: clinical and pathogenesis features.

Neurol. Clin. 2008; 26: pp. 759-780

3. Marchioni Enrico, et al: Acute disseminated encephalomyelitis. Neurol. Sci. 2008; 29: pp. 286-288 
Figure 1. Axial T1 with contrast.

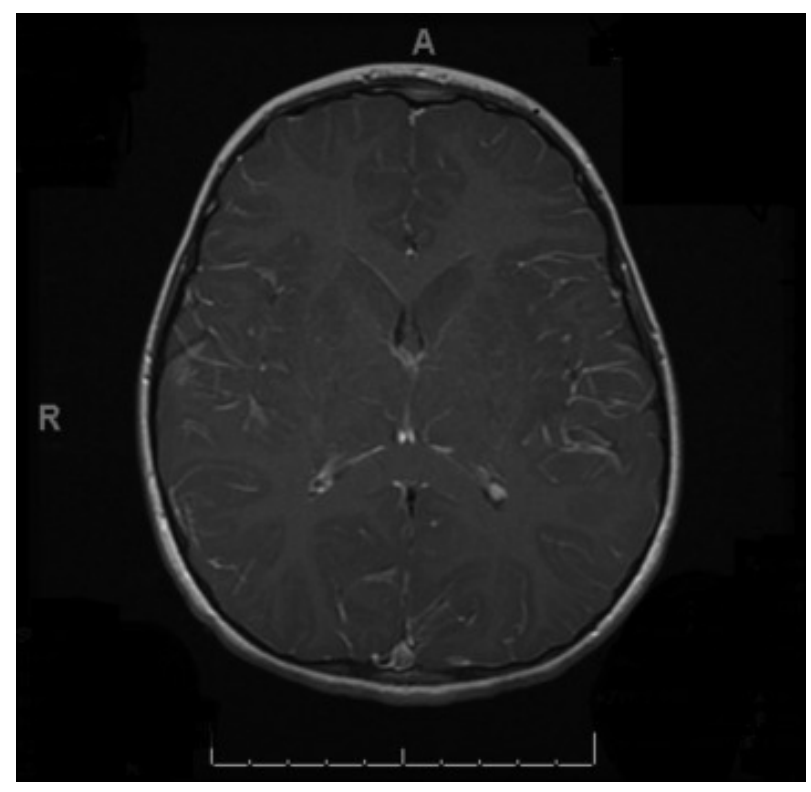


Figure 2. MRI Flair: multiple scattered areas of abnormal T2 hyperintensity involving the white matter of both cerebral hemispheres.

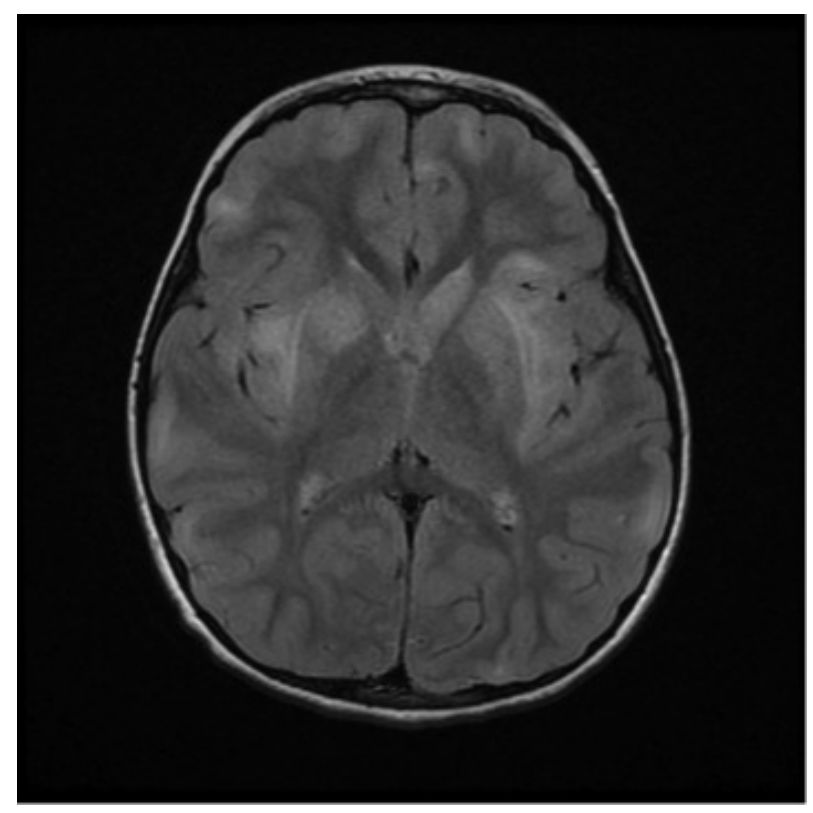


Figure 3. MRI Flair: multiple scattered areas of abnormal T2 hyperintensity involving the white matter of both cerebral hemispheres.

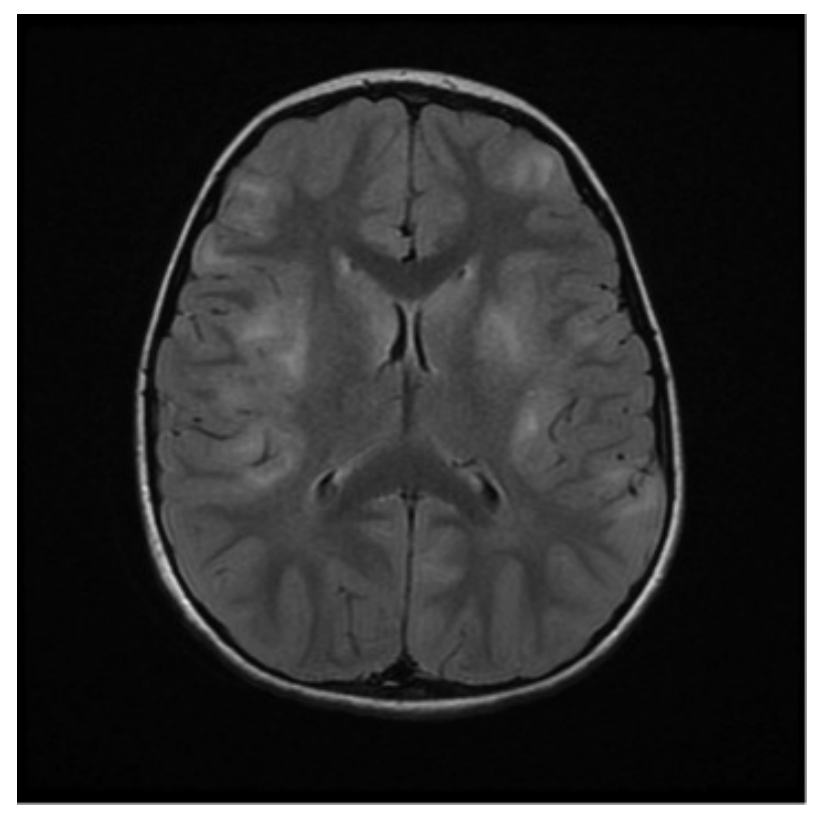

